\section{Germline CEBPA mutation in familial acute myeloid leukemia}

Matilde Boada, ${ }^{1}$ Ana Inés Catalán, ${ }^{2}$ Carolina Ottati, ${ }^{2}$ Florencia Bentancour, ${ }^{1}$ Daniela Lens, ${ }^{2}$ Cecilia Guillermo, ${ }^{1}$ Sofía Grille ${ }^{1,2}$

${ }^{1}$ Hematology Department, and ${ }^{2}$ Basic Medicine Department, Hospital de Clínicas Dr. Manuel Quinela, Montevideo, Uruguay

\begin{abstract}
Myeloid Neoplasms with germline predisposition become part of 2016 World Health Organization (WHO) classification of hematological malignancies since 2016 . CCAAT/enhancer binding protein-alpha (CEBPA) is a myeloid transcription factor located in chromosome 19q. Acute myeloid leukemia (AML) with biallelic mutations of CEBPA AML with recurrent genetic abnormalities according to WHO classification. The inheritance of a germline CEBPA mutation predisposes to the development of AML with autosomal dominant inheritance. Familial CEBPA AML share characteristics with somatic CEBPA AML. However, a higher relapse incidence is reported. We present the case of a 46-years-old male with family history of acute leukemia who was diagnosed with single mutated CEBPA acute myeloid leukemia. The same mutation was found in two of his siblings. The clinical suspicion and proper diagnosis of familial cases is necessary, especially when a related allogenic transplant is indicated in order to select an adequate donor.
\end{abstract}

\section{Introduction}

In 2016, the World Health Organization (WHO) incorporated Myeloid Neoplasm with germline predisposition syndromes into Myelodysplastic Syndromes (MDS) and Acute Myeloid Leukemia (AML) classification. ${ }^{1}$ Estimates suggest that $4-10 \%$ of children and young adults with MDS or AML and $4 \%$ of adults with AML carry inherited mutations affecting cancer susceptibility genes. ${ }^{2}$ Implicated genes according to the WHO classification include: CEBPA, DDX41, RUNX1, ANKRD26, ETV6, GATA2. ${ }^{1}$ However, newer genes have been described more recently. These include SAMD9, SAMD9L, as well as BRCA1 and MSH6 genes more traditionally associated with solid tumors. ${ }^{3}$
CCAAT/enhancer binding proteinalpha (CEBPA) is a myeloid transcription factor expressed in myelomonocytic cells that actively participates in granulocytic maturation. ${ }^{4}$ CEBPA gene is located in chromosome $19 q$ and mutations have been described in 10 to $15 \%$ of AML. ${ }^{5}$ Mutations occur in two main hotspots: N-terminal (frame-shift mutations) and C-terminal (inframe insertions/deletions). ${ }^{6}$

Three different CEBPA mutant patterns can be described in AML: i) Single mutated CEBPA (CEBPAsm), represents $50 \%$ of CEBPA mutated AML, wild type $(w t)$ CEBPA is also expressed; ii) AML double mutated or with biallelic mutation (CEBPAdm), no $w t$ CEBPA expressed; iii) AML with homozygous CEBPA mutation due to loss of heterozygosity (LOH), no $w t$ CEBPA expressed. ${ }^{6}$ AML with biallelic mutations of CEBPA (CEBPAdm) is included as one of AML with recurrent genetic abnormalities in WHO classification. ${ }^{1}$ Moreover, some patients may inherit a germline CEBPA mutation that predisposes to the development of AML with autosomal dominant inheritance. It has been reported that more than $10 \%$ of AML with biallelic CEBPA mutations carried a N-terminal frameshift CEBPA germline mutation, ${ }^{7}$ with acquisition of a C-terminal somatic mutation as a second event in the development of AML.

\section{Case Report}

In February 2020 a 46-year-old male was referred to our Hematology Service at Dr. Manuel Quintela Clinicas Hospital in Montevideo with history of cutaneous bleeding, skin infections and weight loss in the last three months. He worked in a farm and had been exposed to pesticides. He had a complete blood count (CBC) from four months earlier that showed thrombocytopenia $\left(72.0 \times 10^{9} / \mathrm{L}\right)$ and neutropenia $\left(0.34 \times 10^{9} / \mathrm{L}\right)$. At admission white blood cell (WBC) count was $3.0 \times 10^{9} / \mathrm{L}(22 \%$ myeloblasts with Auer Rods), hemoglobin level was $10.2 \mathrm{~g} / \mathrm{dL}$, and platelet count was $42.0 \times 10^{9} / \mathrm{L}$. Bone marrow (BM) aspirate showed $50 \%$ of myeloblasts corresponding to acute myeloblastic leukemia (AML) with maturation according to French American-British (FAB) classification. Blast immunophenotype was: CD34-, CD117++, HLA-DR+, MPO+, CD13+/- with aberrant expression of CD7 and no monocytic, erythroid or megakaryocytic differentiation (Figure 1). Bone marrow biopsy showed $80 \%$ myeloblasts without dysplastic features or
Correspondence: Sofía Grille, Hematology Department, Basic Medicine Department, Hospital de Clínicas Dr. Manuel Quintela, Av. Italia SN, Montevideo, 11600, Uruguay.

Tel.: +59899226612

E-mail: sofiagrille@gmail.com

Key words: Familial leukemia, CEBPA, germline predisposition, acute myeloid leukemia.

Contributions: All authors made substantial contribution to the conception and design and are accountable for all aspects of the work. MB assisted the patient, participated in diagnosis, family assessment and treatment. She wrote this case presentation and reviewed literature. AIC, participated in molecular diagnosis, contributed with writing this case presentation and designed molecular biology images. $\mathrm{CO}$, participated in molecular diagnosis, contributed with writing this case. FB assisted the patient, participated in family assessment and treatment, contributed with writing this case. DL participated in flow cytometry and molecular diagnosis, contributed with writing this case and revised it. $\mathrm{CG}$, participated patient clinical care and in cytomorphology diagnosis, contributed with writing this case and revised it. SG participated patient clinical care as well and molecular and flow cytometry diagnosis, contributed with writing the case and revised it.

Conflict of interest: The authors declare no potential conflict of interest.

Funding: None.

Ethics approval and consent to participate: Written informed consent for publication was obtained from the patient.

Received for publication: 28 February 2021. Revision received: 15 September 2021.

Accepted for publication: 17 September 2021.

This work is licensed under a Creative Commons Attribution-NonCommercial 4.0 International License (CC BY-NC 4.0).

(C) Copyright: the Author(s), 2021

Licensee PAGEPress, Italy

Hematology Reports 2021; 13:9114

doi:10.4081/hr.2021.9114

fibrosis. BM cytogenetics detected an abnormal clone: 48, XY +4 +10 [9] / 46, XY [2]. Molecular analysis demonstrated a monoallelic N-terminal frameshift CEBPA mutation detected by fragment length analysis (25bp deletion) as described by Benthaus et al. ${ }^{8}$ As shown in Figure 1 a deletion of $25 \mathrm{bp}$ was confirmed by sanger sequencing (NG_012022.1:g.531 555del). This CEBPA variant has not been reported 
previously and we have started reporting process with cosmic database. According to European Leukemia NET, patient was classified as Intermediate Risk Acute Myeloid Leukemia.

The patient (proband II-4) had five siblings, four of them were alive. His sister (sibiling II-6) died with 7 years old in 1986 from Acute Leukemia (we do not have the phenotype). His two brothers and one of his sisters were healthy and the other sister (sibiling II-1) had Rheumatoid Arthritis and was receiving biologic treatment. His mother was alive, and his father died at 63-years- old of unknown cause. One of his nieces (relative III-1) was diagnosed with breast cancer at the age of 30-years-old and the other one (relative III-2) died at two-yearsold of an unknown cause (Figure 1). Histocompatibility studies were performed, and the two brothers (sibilings II-3 and II-5) were fully matched. Due to the familial history suggestive of inherited myeloid neoplasia predisposition, the two potential donors (sibilings II-3 and II-5) were studied for CEBPA mutations detection by fragment length analysis and confirmed by sanger sequence and the CEBPA variant present in the patient was found in both cases (Figure 1). Written informed consent were obtained from the patients before evaluation.

Patient received induction with $3+7$ (Idarubicin and cytarabine) not achieving cytomorphologic remission (partial response) after first course (marrow aspirate showed $10 \%$ myeloblasts). A second $3+7$ was delivered as re-induction and then Complete Remission (CR) was achieved. Minimal residual disease (MRD) assessed by multiparametric flow cytometry was high $(0.3 \%)$. The intended treatment was allogenic transplantation. However, his two

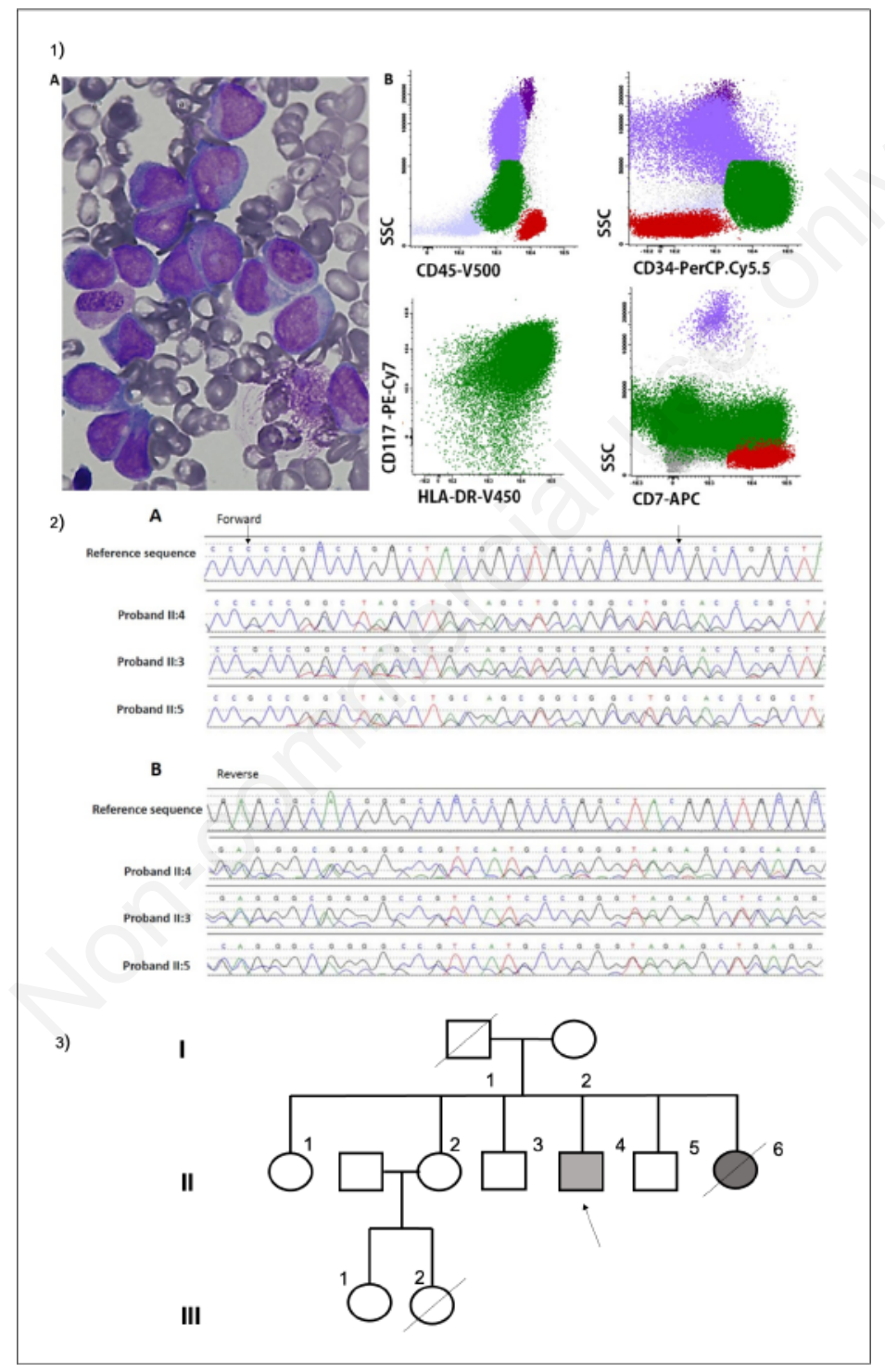

Figure 1. 1) Leukemia Diagnosis. 1.A) Bone marrow aspiration, cytomorphology (May Grunwald Giemsa 100x). 1.B) Immunophenotype. Green: blast cells showing CD34, HLA-DR, CD117 and CD7 expression; Red: T lymphocytes; Grey: erythroblasts; Light purple: neutrophils; Dark purple: Eosinophils. 2) Sequence analysis of CEBPA showing the germline mutation identified in patient II:4 and two brothers II-3 and II-5. 2.A and 2.B shows forward and reverse sequencing respectively. Reference sequence (arrows indicate the beginning and end of the deletion), patient's sequence (Proband II:4) and both siblings (Sibilings II:3 and II:5). This mutation consists of an $25 \mathrm{pb}$ deletion in CEBPA. The normal sequence remains present but its intensity decreased in $50 \%$ and is accompanied by a new signal that correspond to the deleted sequence. 3) Pedigree of the family. Squares refer to males and circles to females. In light grey acute myeloid leukemia; dark grey: acute leukemia of unknown phenotype. An arrow indicates the proband. His father and niece died of unknown cause. GM: germ line mutation. 
related matched donors could not be used because both carried the CEBPA germline mutation. He is now in complete remission, receiving high dose cytarabine consolidation therapy and waiting for an unrelated matched donor.

Written informed consent for publication was obtained from the patient.

\section{Discussion and conclusions}

The first family found to harbor a germline CEBPA mutation was described in United Kingdom in 2004, the index case was a 30-year-old male with de novo M2 AML whose sister developed M2 AML as well only two weeks later. ${ }^{9}$ Both patients exhibited aberrant CD7 expression in myeloblasts. After that, over ten families with Familial CEBPA have been reported with mutations that localize mostly in N-terminal. Median age at diagnosis was reported at 25 years and previous MDS or cytopenia were not common. FAB subtypes were usually M1 and M2 and karyotype was frequently normal. Penetrance was reported to be almost complete. ${ }^{4}$

Clinical presentation of familial CEBPA cases is very similar to somatic CEBPA biallelic AML having similar survival probabilities at diagnosis. Familial CEBPA AML have higher relapse incidence (up to $90 \%$ in some series), with multiple relapses episodes and first relapses presenting later than in sporadic cases. ${ }^{4,10}$ Median time to first relapse in Familial CEBPA AML was reported to be 27 months. ${ }^{11}$ Sensitivity to chemotherapy after relapses is higher in familial cases with better prognosis. ${ }^{11}$ Because of differential molecular profile of subsequent events (different somatic CEBPA mutations), late relapses have been attributed to the novo disease originating from predisposed stem cell pool and that this would explain chemo sensitivity. ${ }^{2}$

Our patient was 46-year-old which is older than the median age at presentation. However, presentation age can range from 2 to 50 -years-old. ${ }^{11}$ In concordance with previous reports, he had M2 AML with CD7 aberrant expression. ${ }^{4}$ Although the majority of familial CEBPA AML present biallelic mutation (CEBPAdm) with N-terminal germline mutation followed by the acquisition of a C-terminal somatic mutation, ${ }^{7}$ a monoallelic $\mathrm{N}$-terminal CEBPA mutation was found in this case. We report a new CEBPA variant. One possible interpretation for monoallelic CEBPA AML is that this germline mutation (first hit) was followed by mutation (second hits) in other not studied genes such as WT1, GATA2, KIT,
TET2, EZH2.$^{10}$ Another possibility is that our patient presented a homozygous mutation due to Loss of heterozigocity (LOH) with no expression of $w t$ CEBPA. This has been reported for CEBPA mutated AML and the underlying proposed mechanism is the deletion of the wild-type allele, or uniparental disomy (UPD) as a result of mitotic recombination. ${ }^{12,13}$ High-resolution single nucleotide polymorphism (SNP) array or next generation sequencing (NGS) allows the detection of UPD. ${ }^{14}$ However, we were not able to confirm LOH as a result of UPD in our patient because these technologies are not available in our country. Another possibility is that CEBPA germline mutation was followed by "second hits" (somatic mutations) in other genes such as WT1, GATA2, KIT, TET2, EZH2. ${ }^{10}$

Although there is no consensus about the requirement of performing allogenic stem cell transplant (allo HSCT) in germline CEBPA AML, our patient did not achieve complete remission after first induction treatment and also have persisted with positive minimal residual disease (MRD) measure by flow cytometry after reinduction. We decided to consolidate with Allo HSCT and he had two HLA matched siblings. However, family background and the presence of patient CEBPA mutation made us to study sibling donors and we found that both carried the same CEBPA variant, thereby becoming non-suitable donors. This case reveals the importance of considering germline testing in patients with CEBPA variants, irrespective of the age of onset.

Lately, next generation sequence (NGS) based platforms for the evaluation of mutation status in AML have become the standard of care in multiple institutions as it is more practical and cost- effective than numerous single-gene assays. Although there are various panels, most of them share a core set of approximately 25 genes frequently mutated in AML which also include several genes that are associated with Myeloid Neoplasms with germline predisposition. ${ }^{1}$ The challenge is to identify which of those would have a possible germline origin. Variant allele frequency (VAF) may provide important information in this regard. It has been reported that mutations in genes with known germline inheritance present at heterozygous range (40-60\%) or homozygous (around 80\%) VAF should trigger further evaluation. ${ }^{15}$ Currently, we are developing an NGS panel for myeloid malignancies at our laboratory that we hope will be soon available soon for AML diagnostic evaluation.

We highlight the importance of familial AML suspicion based on family history, especially when a related donor transplant is planned. Algorithms has been described in order to guide the study of a suspect myeloid neoplasm with germline predisposition syndrome. ${ }^{16}$ Our patient presented a first degree relative with acute leukemia at a young age onset and a CEBPA mutation. This leads us to consider the possibility of a CEBPA familial AML. The diagnosis is not only beneficial for the patient, allowing a suitable donor selection, but also to asymptomatic affected carriers in order to develop an adequate surveillance strategy. ${ }^{15,16}$

\section{References}

1. Arber DA, Orazi A, Hasserjian R, et al. The 2016 revision to the World Health Organization classification of myeloid neoplasms and acute leukemia. Blood 2016;127:2391-405.

2. Churpek JE. Familial myelodysplastic syndrome/acute myeloid leukemia. Best Pract Res Clin Haematol 2017;30:2879.

3. University of Chicago Hematopoietic Malignancies Cancer Risk Team. How I diagnose and manage individuals at risk for inherited myeloid malignancies. Blood 2016;128:1800-13.

4. Tawana K, Rio-Machin A, Preudhomme C, Fitzgibbon J. Familial CEBPA-mutated acute myeloid leukemia. Semin. Hematol 2017;54:8793.

5. Snaddon J, Smith ML, Neat M, et al. Mutations of CEBPA in acute myeloid leukemia FAB types M1 and M2. Genes, Chromosomes Cancer 2003;37:72-8.

6. Fasan A, Haferlach C, Alpermann T, et al. The role of different genetic subtypes of CEBPA mutated AML. Leukemia 2014;28:794-803.

7. Pabst T, Eyholzer M, Haefliger S, et al. Somatic CEBPA mutations are a frequent second event in families with germline CEBPA mutations and familial acute myeloid leukemia. J Clin Oncol 2008;26:5088-93.

8. Benthaus T, Schneider F, Mellert G, et al. Rapid and sensitive screening for CEBPA mutations in acute myeloid leukaemia. $\mathrm{Br} \mathrm{J}$ Haematol 2008;143:230-9.

9. Smith ML, Cavenagh JD, Lister TA, Fitzgibbon J. Mutation of CEBPA in familial acute myeloid leukemia. N Engl J Med 2004;351:2403-7.

10. Brown AL, Hahn CN, Scott HS. Secondary leukemia in patients with germline transcription factor mutations 
(RUNX1, GATA2, CEBPA). Blood 2020;136:24-35.

11. Tawana K, Wang J, Renneville A, et al. Disease evolution and outcomes in familial AML with germline CEBPA mutations. Blood 2015;126:1214-23.

12. Wouters BJ, Sanders MA, Lugthart S, et al. Segmental uniparental disomy as a recurrent mechanism for homozygous CEBPA mutations in acute myeloid leukemia. Leukemia 2007;21:2382-4.

13. Fitzgibbon J, Smith LL, Raghavan M, et al. Association between acquired uniparental disomy and homozygous gene mutation in acute myeloid leukemias. Cancer Res 2005;65:9152-4.

14. Yamazawa K, Ogata T, Ferguson-Smith AC. Uniparental disomy and human disease: an overview. Am J Med Genet C Semin Med Genet 2010;154C:329-4.

15. DiNardo CD, Bannon SA, Routbort M, et al. Evaluation of Patients and Families With Concern for Predispositions to Hematologic
Malignancies Within the Hereditary Hematologic Malignancy Clinic (HHMC). Clin Lymphoma Myeloma Leuk 2016;16:417-28.

16. Churpek JE, Lorenz R, Nedumgottil S, et al. Proposal for the clinical detection and management of patients and their family members with familial myelodysplastic syndrome/acute leukemia predisposition syndromes. Leuk Lymphoma 2013;54:28-35. 